\title{
Case of early-stage gastric cancer identified in the gastric mucosa with lanthanum phosphate deposition
}

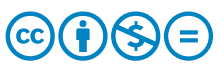

\author{
Authors \\ Institutions \\ 1 Department of Gastroenterology, Nagaoka Red Cross \\ Hospital, Nagaoka, Japan \\ 2 Department of Pathology, Nagaoka Red Cross Hospital, \\ Nagaoka, Japan \\ 3 Division of Gastroenterology and Hepatology, Graduate \\ School of Medical and Dental Sciences, Nigata \\ University, Niigata, Japan
}

Masafumi Takatsuna ${ }^{1}$, Manabu Takeuchi ${ }^{1}$, Hiroyuki Usuda ${ }^{2}$, Shuji Terai ${ }^{3}$

submitted 31.10 .2018

accepted after revision 21.2.2019

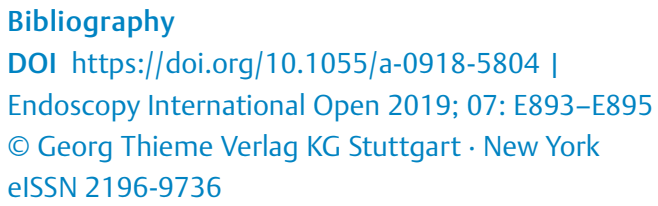

\section{ABSTRACT}

Background and study aims A 78-year-old man with $\mathrm{He}$ licobacter pylori infection had been undergoing hemodialysis for chronic renal failure and receiving lanthanum carbonate preparations for 3 years. Endoscopy revealed fine white granular discoloration throughout the stomach, a moderately reddish depression on the lesser curvature of the gastric angle, and white granular discoloration in the surrounding area. A magnified image using narrow-band imaging showed that the depressed part had irregular vascular and pit structures. We established a diagnosis of intramucosal gastric cancer and performed endoscopic submucosal dissection. Histopathological examination revealed a well-differentiated adenocarcinoma that was confined to the mucosa of the depressed area. Moreover, using an electron probe microanalyzer-equipped electron microscope, we found that the degree of lanthanum deposition was lower in the tumor region than in the non-tumor region. Thus, the current case can help in understanding the relationship between lanthanum deposition and early-stage gastric cancer. Because gastric cancers can occur in lanthanum deposit-containing mucosa, esophagogastroduodenoscopy should be used carefully after understanding the characteristics of early- stage gastric cancer in such cases.

\section{Introduction}

Patients with end-stage renal disease require phosphate binders to trap dietary phosphate. Lanthanum carbonate, an orally administered phosphate binder, is used for treating renal failure. Some reports have described lanthanum carbonate deposition in the gastric mucosa $[1,2]$. We reported a patient with early-stage gastric cancer accompanied with lanthanum phosphate deposition in the gastric mucosa.

\section{Case report}

A 78-year-old man with Helicobacter pylori infection was undergoing hemodialysis for chronic renal failure and was receiving lanthanum carbonate preparations for 3 years. The patient underwent esophagogastroduodenoscopy (EGD) during a health checkup. The endoscopic view revealed fine white granular dis- coloration throughout the stomach ( $\boldsymbol{F}$ Fig.1a), a moderately reddish depression (diameter $10 \mathrm{~mm}$ ) on the lesser curvature of the gastric angle, and white granular discoloration in the surrounding area ( $\mathbf{F i g} . \mathbf{1}$ b and $\boldsymbol{\nabla}$ Fig. $\mathbf{1 c}$ ). Magnified narrow-band imaging showed a fine microvascular network pattern in the depressed part; surface pattern was mostly absent ( $\mathbf{F i g . 1 d ) . ~}$ Biopsies were performed and the lesion was histopathologically diagnosed as well-differentiated tubular adenocarcinoma. These characteristic findings had not been detected during the previous endoscopic examination. We established a diagnosis of intramucosal gastric cancer and performed endoscopic submucosal dissection (ESD) after 2 months. Histopathological results revealed that the well-differentiated tubular adenocarcinoma was confined to the mucosa of the depressed area ( $\triangleright$ Fig.2a). Moreover, using an electron probe microanalyzerequipped electron microscope, we detected weak signals for lanthanum deposition in the tumor tissue ( $>$ Fig. 2b); a higher 


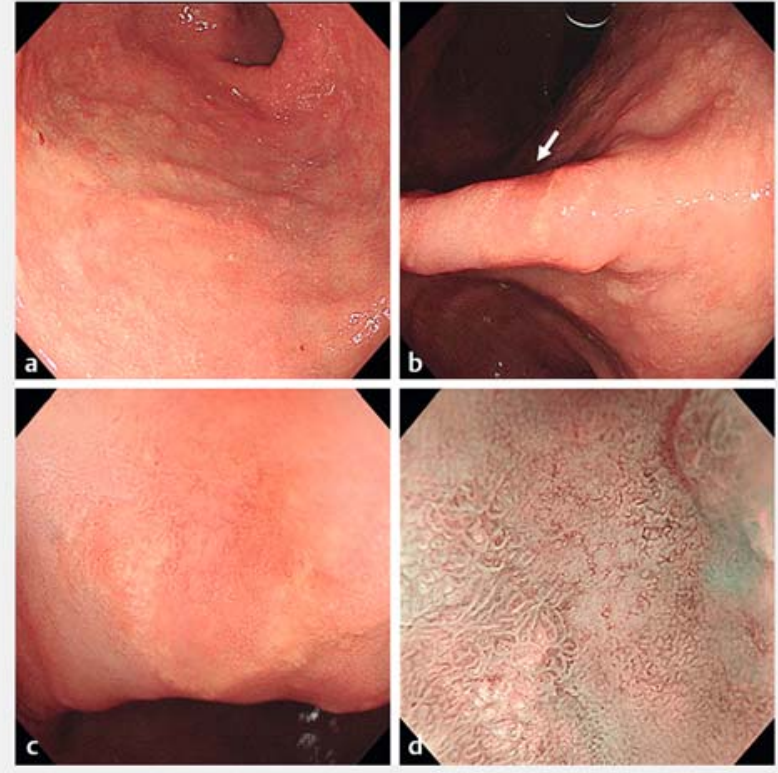

- Fig. 1 a Gastric mucosa observed under white light, b depressed lesion on the lesser curvature of the gastric angle (white arrow), c magnified image of the depressed lesion, and $\mathbf{d}$ magnified image using narrow-band imaging (NBI).

degree of lanthanum deposition was observed in the non-tumor region than in the tumor tissue ( $\boldsymbol{F i g . 2 c}$ ). Immunohistochemical staining showed CD68-positive cells in the non-tumor region, indicating presence of macrophages that had phagocytosed lanthanum ( $\triangleright$ Fig. 3 ).

\section{Discussion}

Endoscopic findings of lanthanum deposition in the gastric mucosa are reportedly described as numerous small and irregular white spots and annular white thickening [1,2], consistent with the findings in the current case.

We identified a well-differentiated tubular adenocarcinoma presenting as a depressed area in the lanthanum deposit-containing mucosa. Tabuchi et al reported that early gastric cancer (papillary adenocarcinoma) with lanthanum deposition was observed as a depressed area on endoscopy [3]. Our case showed findings similar to the findings of this report.

We found a lower degree of lanthanum deposition in the tumor area than in the non-tumor area. Whether lanthanum deposition is a result of mucosal damage by $H$. pylori infection remains controversial [4-6], but some reports have suggested an association between mucosal distribution of lanthanum deposition and intestinal metaplasia [2,7]. In gastric intestinal metaplasia, expression of claudin, a major protein of tight junctions in the epithelium that play an important role in fence function, is reported to differ from that in gastric cancer [8]. We hypothesized that this difference could lead to a difference in lanthanum deposition or absorption in the gastric mucosa. This may be why distribution of lanthanum deposits is different

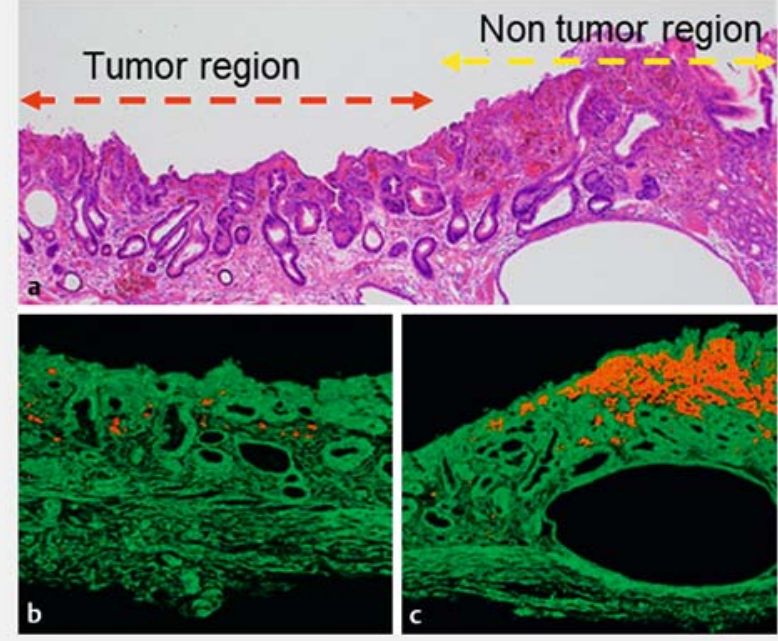

- Fig. 2 a Histopathological image of the endoscopic submucosal dissection. The part marked with the red line is the region of welldifferentiated tubular adenocarcinoma, whereas the part marked with the yellow line is the non-tumor region. b Analysis using electron probe microanalyzer $\mathbf{c}$ revealed a negligible amount of lanthanum deposition in the tumor region and a high degree of lanthanum deposition (orange) in the non-tumor region.

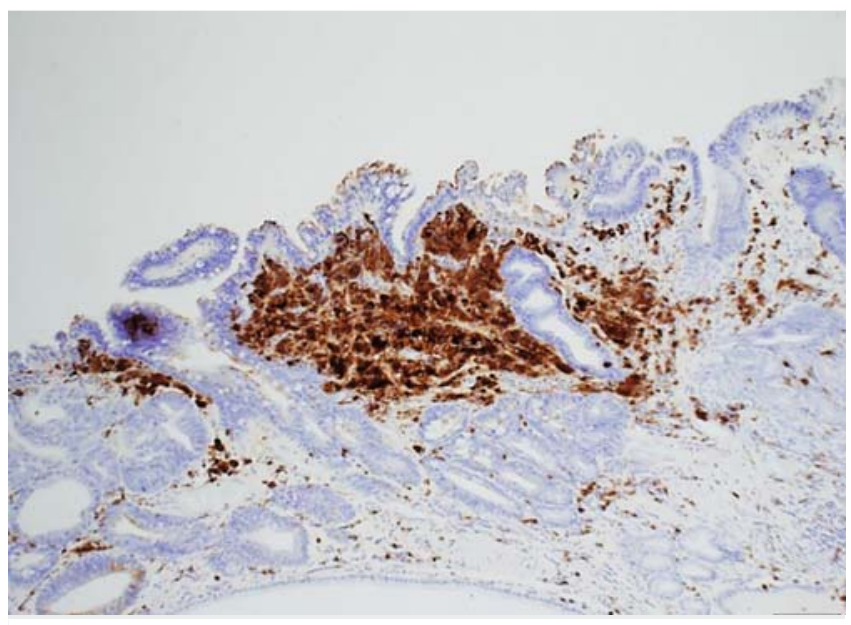

- Fig.3 CD68-positive macrophages in the area with lanthanum deposits.

between tumor and non-tumor regions. Our case is very rare, and this report would add to the literature because lanthanum-induced differences between the tumor and non-tumor areas were clearly distinguishable in our patient.

\section{Conclusion}

The current case is of interest, as it can help in understanding the relationship between lanthanum deposition and earlystage gastric cancer. It is important to accumulate reports from a number of similar cases and to examine the endoscopic 
imaging findings of early-stage gastric cancer in the gastric mucosa with lanthanum deposition.

Competing interests

None

\section{References}

[1] Iwamuro M, Kanzaki H, Tanaka T et al. Lanthanum phosphate deposition in the gastric mucosa of patients with chronic renal failure. Nihon Shokakibyo Gakkai Zasshi 2016; 113: 1216 - 1222

[2] Makino M, Kawaguchi K, Shimojo $\mathrm{H}$ et al. Extensive lanthanum deposition in the gastric mucosa: The first histopathological report. Pathol Int 2015; 65: $33-37$
[3] Tabuchi M, Takeshima F, Akazawa Y et al. Early gastric cancer with diffuse lanthanum deposition. Intern Med 2019; 58: 447 - 448

[4] Ban S, Suzuki S, Kubota K et al. Gastric mucosal status susceptible to lanthanum deposition in patients treated with dialysis and lanthanum carbonate. Ann Diagn Pathol 2017; 26: 6 - 9

[5] Shitomi Y, Nishida H, Kusaba T et al. Gastric lanthanosis (lanthanum deposition) in dialysis patients treated with lanthanum carbonate. Pathol Int 2017; 67: 389-397

[6] Iwamuro M, Urata $\mathrm{H}$, Tanaka T et al. Lanthanum deposition in the stomach in the absence of Helicobacter pylori Infection. Intern Med 2018; 57: $801-806$

[7] Tonooka A, Uda S, Tanaka H et al. Possibility of lanthanum absorption in the stomach. Clin Kidney J 2015; 8: 572-575

[8] Oshima T, Shan J, Okugawa T et al. Down-regulation of claudin-18 is associated with the proliferative and invasive potential of gastric cancer at the invasive front. PLoS One 2013; 8: e74757 